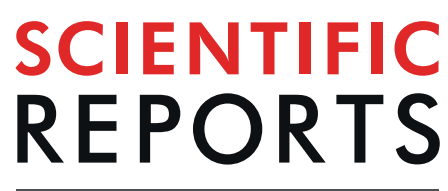

natureresearch

\title{
OPEN Predicting novel genomic regions linked to genetic disorders using GWAS and chromosome conformation data - a case study of schizophrenia
}

\begin{abstract}
Daniel S. Buxton, Declan J. Batten, Jonathan J. Crofts $(\mathbb{D})$ \& Nadia Chuzhanova $\mathbb{D}^{*}$
Genome-wide association studies identified numerous loci harbouring single nucleotide polymorphisms (SNPs) associated with various human diseases, although the causal role of many of them remains unknown. In this paper, we postulate that co-location and shared biological function of novel genes with genes known to associate with a specific phenotype make them potential candidates linked to the same phenotype ("guilt-by-proxy"). We propose a novel network-based approach for predicting candidate genes/genomic regions utilising the knowledge of the 3D architecture of the human genome and GWAS data. As a case study we used a well-studied polygenic disorder - schizophrenia - for which we compiled a comprehensive dataset of SNPs. Our approach revealed 634 novel regions covering $\sim 398 \mathrm{Mb}$ of the human genome and harbouring $\sim 9000$ genes. Using various network measures and enrichment analysis, we identified subsets of genes and investigated the plausibility of these genes/ regions having an association with schizophrenia using literature search and bioinformatics resources. We identified several genes/regions with previously reported associations with schizophrenia, thus providing proof-of-concept, as well as novel candidates with no prior known associations. This approach has the potential to identify novel genes/genomic regions linked to other polygenic disorders and provide means of aggregating genes/SNPs for further investigation.
\end{abstract}

Decades long genome-wide association studies (GWASs) have identified numerous loci harbouring single nucleotide polymorphisms (SNPs) and other genetic variants associated with a range of human diseases, although the causal role of many of them remains unknown. In silico ascertainment of SNPs and SNP-harbouring loci is hampered by several factors including their location and small effect size of SNPs. It is known that approximately $93 \%$ of disease-associated variants reside outside protein coding regions ${ }^{1}$, potentially within unknown regulatory elements. These regulatory elements do not necessarily target the nearest gene(s) in the vicinity but may reside at considerable distances from the genes they regulate [reviewed in ref. ${ }^{2}$ ]. Indeed, it was recently shown that only $14 \%$ of SNPs in non-coding regions target nearest genes ${ }^{3}$, prompting a need for more accurate ways of identifying SNP-target gene pairs, instead of a simple assignment of a SNP to the nearest gene. In addition, many diseases are polygenic, relying on the cooperation of small effect size SNPs in more than one gene, in order for the disease phenotype to exist. Identification of these relevant sets of genes/SNPs and, most importantly, providing a plausible biological explanation for their cooperation is not a trivial undertaking. SNPs are usually aggregated either at the level of genes or a set of genes which share a known biological function(s) or pathway. To assess the joint effect of groups of SNPs, various set-based approaches, not requiring individual genotype data, have been developed (e.g. ref. $\left.{ }^{4}\right)$. Another group of methods is based on polygenic risk scores ${ }^{5}$ that are usually used to predict phenotype likelihood by assessing the joint effect of a group of SNPs. The latter approaches usually require two samples - a discovery sample, comprising GWAS summary statistics, and an independent target sample with known individual genotype data, which may not be readily available. 
In this paper we postulate that co-location of novel genes with genes, known to be associated with a specific phenotype, and their enrichment in the same biological function or pathway as known genes, make them good candidates for novel genes, linked to the same phenotype ("guilt-by-proxy"). We hypothesise that SNPs residing within these groups of co-located genes, comprising both novel and known "guilty" genes, may contribute to the observed phenotype either individually (when a single common SNP residing in one of these genes could cause a phenotype), or collectively (when SNPs residing in several functionally-related genes may have an additive effect on the observed phenotype), or selectively (when SNPs exhibit genome-wide significance only in a smaller and possibly more homogeneous subgroup of patients stratified by their origin, age, gender, etc.). We further postulate that both non-coding SNPs and their potential target genes also reside within co-located loci.

To find novel genomic regions co-located with loci that have proven association with a given phenotype, we propose a conceptually new approach to identification of these co-located regions that utilises the knowledge of the $3 \mathrm{D}$ architecture of the human genome and is based on in silico analysis of networks of co-located loci. As a case study for the proposed approach, we investigate the well-studied disorder - schizophrenia (SCZ). We further expanded a dataset of SNPs generated by the Psychiatric Genomics Consortium (PGC; refs. ${ }^{6,7}$ ) by SNPs found via review of literature and the use of various bioinformatics resources. As a result, 346 genes linked to schizophrenia and 1,252,901 SNPs were identified although not a single SNP was found to have a large enough effect size to claim sole responsibility for SCZ development. Hence, SCZ is considered to be a polygenic disorder that relies on the cooperation of small effect size SNPs in order for the disease phenotype to exist ${ }^{8}$.

To assess the co-location (or proximity) of two distinct loci, not necessarily on the same chromosome, we used datasets of interaction frequencies generated by various chromosome conformation capture techniques ${ }^{9-11}$ and available for a range of cell lines. Note that the frequency of intra- and inter-chromosomal interactions between two loci is inversely proportional to their closeness within the cell nucleus.

We used brain-specific expression quantitative trait loci (eQTL) data from Braineac/Brain eQTL Almanac database $^{12}$ to choose the most relevant cell line, which emulates 3D interactions in brain tissues and for which chromosome conformation data was available at the time when this study was initiated. A network of interactions was created from the interaction data for this cell line with nodes representing genomic regions, connected by edges, weighted by interaction frequencies. We calculated various network centrality (e.g. degree) measures ${ }^{13}$ and identified communities (i.e. densely connected subnetworks) existing within the network with the aim of revealing influential nodes/regions and close-knit communities within SCZ interaction networks. In the subsequent analysis of candidate regions and subnetworks, we used functional annotation and enrichment analysis tools provided by the Gene Ontology Consortium ${ }^{14,15}$, functional annotation and enrichment analysis tools DAVID ${ }^{16}$, ENCODE data ${ }^{17}$ and compared our results with results obtained by recent SCZ GWAS ${ }^{18}$ and machine-learning approach $^{19}$ to build a compelling case for the phenotypic role of newly found loci that were not present in the expanded PGC dataset but were found solely on the basis of their close proximity to, and shared function with, the "guilty" genes.

\section{Results}

Selection of the cell line enriched in brain-related eQTL. To determine which cell line most closely resembled interactions in brain tissues, brain eQTL data ${ }^{12}$ was used. The majority $(>95 \%)$ of cis-eQTL pairs recorded in Braineac/Brain eQTL Almanac database ${ }^{12}$ were found residing within fragments that interacted with frequency $>0$ according to in situ $\mathrm{Hi}-\mathrm{C}$ data ${ }^{10}$ for all cell lines and bin sizes. However, the proportion of ciseQTL pairs residing within strongly interacting fragments (with frequency $\geq 64$, corresponding to $\sim 0.05 \%$ of the strongest interactions in the datasets of normalised counts) varied between cell lines. The highest proportion of strong cis-eQTL pairs $\left(\mathrm{p} \leq 10^{-6}\right)$ among these strongly interacting fragments was found for the GM12878 cell line ( $84 \%)$, followed by IMR90 (46\%) and HMEC (45.6\%). Fisher's exact test confirmed that there is a significant $\left(\mathrm{p}<10^{-398}\right)$ enrichment of intra-chromosomal interactions in brain-related eQTL signals for the GM12878 cell line. Moreover, the comparison with the $\mathrm{Hi}-\mathrm{C}$ data for adult brain cells that recently became available via PsychENCODE resources ${ }^{19}$ showed that $\sim 70 \%$ of interactions in brain cells are preserved in the GM12878 cell line. Therefore, GM12878 was clearly the most suitable choice of cell line for emulating 3D interactions in brain tissues. Hence, subsequent approaches utilised only Hi-C data for the GM12878 cell line.

Properties of original and extended SCZ interaction networks. The SCZ networks of interactions between the extended regions of the $346 \mathrm{SCZ}$-associated genes (Supplementary Table S1) and/or their regulatory regions amalgamated into 103 non-overlapping extended genomic regions (EGRs), were created as described in Materials and Methods. Only inter-chromosomal interaction with frequency $\geq 64$, corresponding to $\sim 0.05 \%$ of the strongest interactions in the datasets of counts normalised using Knight and Ruiz matrix balancing procedure, were considered. As a result, 32 EGRs corresponding to 32 SCZ-associated genes (see Supplementary Table S2) from the set of $346 \mathrm{SCZ}$-associated genes did not show inter-chromosomal interactions to any other regions; therefore, these isolated nodes were not used in the subsequent analyses. One $100 \mathrm{~K}$ region on chromosome 1 (chr1:121,400,001-121,500,000) was found to interact with almost every other $100 \mathrm{~K}$ regions. This is a gene-less region which was only partially present in the earlier NCBI36/hg18 assembly. For these reasons the region and all interactions with this region were deleted from the dataset of interactions.

The original network is disconnected and consists of 34 subnetworks (Supplementary Fig. S1). Together with 61 regions harbouring 285 SCZ-associated genes and their regulatory elements, this network contains 85 new regions that exhibit strong interactions with regions harbouring SCZ-associated genes but resided outside of those 108 SCZ risk loci identified by GWAS studies ${ }^{7}$. Comparison of regions corresponding to the nodes in the original network with loci identified by the recent GWAS (ref. ${ }^{18}$ and Supplementary Table 4 therein) shows that 58 out of the 145 non-overlapping loci identified by meta-analysis of CLOZUK and other available up-to-date PGC GWAS datasets as harbouring significant $\left(\mathrm{p}<5 \times 10^{-8}\right)$ SNPs were also present in the original network. Two 
loci on chromosome $\mathrm{X}$ were not found since all loci residing within this chromosome were excluded from the list of 346 genes. Further, 123 and 89 out of 346 SCZ-associated genes were also found in, respectively, the SCZ high-confidence and risk gene lists (http://resource.psychencode.org) reported in ref. ${ }^{19}$.

The extended network comprises 700 nodes (regions), covering $419 \mathrm{Mb}$ of the human genome and comprising 71 regions harbouring 314 SCZ-associated genes and 629 novel regions harbouring 8,984 genes. All the 143 loci identified in ref. ${ }^{18}$ (with the exception of regions on chromosome $\mathrm{X}$ as explained above) were present in the extended network. Out of the 302 genes listed in the SCZ high-confidence gene list ${ }^{19}, 117$ genes were found to reside within novel regions, whereas out of the 606 genes listed in the SCZ risk gene list ${ }^{19}$ (without genes overlapping with the SCZ high-confidence gene list), 337 genes were in common.

The extended network consists of one giant component (666 nodes) and 13 disconnected subnetworks (Supplementary Fig. S2), comprising two to six nodes. Two nodes in the giant component, corresponding to regions chr22:38,800,001-43,700,000 (labelled as 22:389-437 in $100 \mathrm{~Kb}$ scale) and chrX:20,500,001-21,900,000 (labelled as 23:204-219), have the highest degree, respectively 328 and 304. The region on chromosome 22 together with all connected regions harbours 5513 genes (Supplementary Table S3). A total of 105 genes were enriched in the biological processes GO term "regulation of neuron death" (enrichment score 1.57; false discovery rate, $\mathrm{FDR}=0.0179 ; \mathrm{p}$-value after Bonferroni correction for multiple testing 0.00175); and 220 genes were enriched in the GO term "chromatin organization" (enrichment score 1.41, FDR $=0.00443, \mathrm{p}=0.00118$ ). The region on chromosome X together with all neighbours of this region harbour 7200 genes (Supplementary Table S4); 580 genes were enriched in the GO cellular component term "neuron part" (enrichment score 1.17, FDR $=0.00191$, p > 0.05); 514 genes were enriched in GO biological processes term "response to oxygen-containing compound" (enrichment score 1.2, FDR $=0.0317, \mathrm{p}>0.05$ ).

A total of $31 \mathrm{SCZ}$-associated genes were found to reside within 13 disconnected subnetworks, present both in the original and extended networks, with approximately $2 \%$ (2176) of all SNPs residing within these genes. Although none of these SNPs reached the genome-wide significance level as recorded in the expanded PGC dataset used, recent studies ${ }^{18}$ performed on a bigger cohort of patients/controls identified significant SNPs in regions comprising ten disconnected subnetworks (clusters 7-9, 12-13, 15, 17-19 and 22 in Fig. 1 and Supplementary Table S5).

Communities in the extended network. We identified seven communities (clusters) in the giant component of the extended network (Figs. 1-3). Larger communities have been further subdivided into smaller subcommunities (e.g. subcommunity 4 of community 1 denoted as 1.4 and so on) with the aim to find more densely connected and co-located subnetworks of genes enriched in a specific biological function and explore the role these functionally-related genes may play in the observed phenotype. A tabular representation of communities together with complete sets of genes residing within nodes comprising each community is given in Supplementary Table S5. The number of nodes in the selected communities/subcommunities and genes enriched in various $\mathrm{GO}$ terms are summarised in Table 1.

From the genes residing within regions comprising five largest components (clusters 1-5) of the extended network, several groups of genes with significant enrichment in GO terms associated with various biological processes, pathways, reactome pathways and protein classes emerged. The highest fold enrichment $(\mathrm{FDR}<0.05)$ was observed for "regulation of complement cascade" (31.81), "DNA demethylation" (13.43) and "DNA methylation" (10.72) (see Table 1).

\section{Discussion}

In this study we used a new network-based approach for identification of novel genes/loci associated with a specific phenotype that takes into account the co-location and shared biological function of newly found genes with so-called "guilty" genes, i.e. genes that are known to associate with the phenotype in question. Using schizophrenia as a case study we compiled a dataset of 346 "guilty" genes identified by early PGC GWAS ${ }^{6,7}$ but expanded with genes/ SNPs identified via a comprehensive literature search. In the resulting dataset, 212 of "guilty" genes were in common with the recently compiled lists of high-confidence and SCZ risk genes ${ }^{19}$ (http://resource.psychencode.org). Moreover, 58 loci were found to be in common between loci harbouring 346 genes and 145 loci identified by a recent meta-analysis as harbouring significant SCZ-associated $\mathrm{SNPs}^{18}$.

Using non-brain specific $\mathrm{Hi}-\mathrm{C}$ interaction data for the GM12878 cell line, which preserves $~ 70 \%$ interactions recorded in adult brain cells that have recently become available via PsychENCODE resources ${ }^{19}$, we created an original network of interactions between regions harbouring those 346 "guilty" genes and extended it by adding regions that have strong inter-chromosomal interactions with the nodes/regions from the original network. All the 143 loci identified in ref. ${ }^{18}$ were present in the extended network. Out of the 302 and 606 genes listed, respectively, in high-confidence and SCZ risk gene lists ${ }^{19}, 80 \%$ and $70 \%$ of genes were found to reside within the regions comprising the extended network created using non-brain $\mathrm{Hi}-\mathrm{C}$ data. These rather high overlaps show the potential of the proposed approach to identify novel SCZ-related genes. Note that our approach is solely based on co-location and shared biological function of newly found genes with the "guilty" genes. These percentages, on the other hand, may reflect the fact that only $70 \%$ of brain-specific interactions are preserved in the karyotypically normal B lymphoblastoid cell line GM12878 used in this study.

In several examples below, we discuss the plausibility of genes/SNPs residing within various important (from network science point of view) nodes or clusters found contributing to SCZ either individually or selectively. In the absence of individual genotype data, the assessment of cumulative effect of several SNPs is purely speculative and needs further investigation.

Important nodes. Two nodes in the extended network corresponding to regions chr22:38,800,00143,700,000 and chrX:20,500,001-21,900,000 were found to be of possible importance on the basis of the high 


\begin{tabular}{|c|c|c|c|c|}
\hline Community & \begin{tabular}{|l|} 
Total number of \\
nodes/length $(\mathrm{Mb})$ in \\
(sub)community
\end{tabular} & Enrichment in GO term ${ }^{\dagger}$ & p-value ${ }^{*}$ & List of genes \\
\hline 1 & $176 / 110.9$ & $\begin{array}{l}\text { Alzheimer disease- } \\
\text { presenilin pathway } \\
\text { (GOR) }\end{array}$ & $2.24 \times 10^{-2}$ & $\begin{array}{l}\text { WNT8A, NOTCH3 (S,N,P) }{ }^{\S}, M M P 9(\text { AD, S,N,P), PCSK4, CTNNA1, JUP, TCF3, } \\
\text { HN1, FZD2 (P), WNT9B, MMP25, MMP24 (P), WNT3 (PD,N), ERN1, LRP5, } \\
\text { LRP3, PSENEN (AD), KAT7, ERN2, ACTG1, APH1A, MMP25, APH1B (AD,N), } \\
\text { APBB3 }\end{array}$ \\
\hline 1.1 & $5 / 3.7$ & oxygen transport (GOR) & $1.48 \times 10^{-2}$ & $H B A 2, H B A 1, H B Z, H B M, H B Q 1$ \\
\hline \multirow[t]{3}{*}{1.2} & \multirow[t]{3}{*}{$40 / 51.3$} & $\begin{array}{l}\text { homophilic cell adhesion } \\
\text { via plasma membrane } \\
\text { adhesion molecules } \\
\text { (GOR) }\end{array}$ & $3.73 \times 10^{-21}$ & $\begin{array}{l}\text { BSG, CDH22, CLSTN1, } \\
\text { MYOT, PCDHA1, PCDHA10, PCDHA11, } \\
\text { PCDHA12, PCDHA13, } \\
\text { PCDHA2, PCDHA3, } \\
\text { PCDHA4, PCDHA5, } \\
\text { PCDHA6, PCDHA7, } \\
\text { PCDHA8, PCDHA9, } \\
\text { PCDHAC1, PCDHAC2, } \\
\text { PCDHB1, PCDHB10, } \\
\text { PCDHB11, PCDHB12, } \\
\text { PCDHB13, PCDHB14, } \\
\text { PCDHB15, PCDHB16, } \\
\text { PCDHB18P, PCDHB2, } \\
\text { PCDHB3, PCDHB4, } \\
\text { PCDHB5, PCDHB6, } \\
\text { PCDHB7, PCDHB8, } \\
\text { PCDHB9, PCDHGA1, } \\
\text { PCDHGA10, PCDHGA11, } \\
\text { PCDHGA12, PCDHGA2, } \\
\text { PCDHGA3, PCDHGA4, } \\
\text { PCDHGA5, PCDHGA6, } \\
\text { PCDHGA7, PCDHGA8, } \\
\text { PCDHGA9, PCDHGB1, } \\
\text { PCDHGB2, PCDHGB3, } \\
\text { PCDHGB4, PCDHGB5, } \\
\text { PCDHGB6, PCDHGB7, } \\
\text { PCDHGC3, PCDHGC4, } \\
\text { PCDHGC5, PVRL2 }\end{array}$ \\
\hline & & $\begin{array}{l}\text { vasopressin synthesis } \\
\text { pathway (GOR) }\end{array}$ & $9.03 \times 10^{-7}$ & $H N 1, M M P 24(\mathbf{P}), L R P 5$ \\
\hline & & $\begin{array}{l}\text { Neuropeptide protein } \\
\text { class (GOR) }\end{array}$ & $3.05 \times 10^{-3}$ & $\begin{array}{l}\text { OXT }(\mathbf{P}), A P I T D 1, \\
P D Y N(\mathbf{S}, \mathbf{N}, \mathbf{P}), P P Y, \\
P Y Y(P), C O R T, A V P(\mathbf{P}), G H R H(\mathbf{N}, \mathbf{P})\end{array}$ \\
\hline \multirow{3}{*}{1.3} & \multirow{3}{*}{$31 / 22.2$} & $\begin{array}{l}\text { histone protein class } \\
\text { (GOR) }\end{array}$ & $6.32 \times 10^{-8}$ & $\begin{array}{l}\text { HIST1H2BN, HIST1H2AI, } \\
\text { HIST1H2BM, HIST1H2AM, } \\
\text { HIST1H2AL, HIST1H2AI, } \\
\text { HIST1H2AK, HILS1, } \\
\text { HIST2H2AC, HIST2H2BC, } \\
\text { HIST1H2AJ, HIST1H2BL, } \\
\text { HIST1H1B, HIST2H2AA3, } \\
\text { HIST2H2AA4, HIST2H2BE, } \\
\text { HIST2H4A, HIST2H4B, } \\
\text { HIST1H4K, HIST1H4L, } \\
\text { HIST1H4J, HIST1H2BO, } \\
\text { HIST2H2AB }\end{array}$ \\
\hline & & histone fold (DAVID) & $4.6 \times 10^{-12}$ & $\begin{array}{l}\text { HIST1H2AI, HIST1H2AJ, HIST1H2AK, HIST1H2AL, } \\
\text { HISTIH2AM, HIST1H2BL, } \\
\text { HIST1H2BM, HIST1H2BN, } \\
\text { HIST1H2BO, HIST1H3H, HIST1H3I, HIST1H3J, } \\
\text { HIST1H4J, HIST1H4K, } \\
\text { HIST1H4L, HIST2H2AA3, } \\
\text { HIST2H2AA4, HIST2H2AB, } \\
\text { HIST2H2AC, HIST2H2BC, } \\
\text { HIST2H2BE, HIST2H3A, } \\
\text { HIST2H3C, HIST2H4A, } \\
\text { HIST2H4B }\end{array}$ \\
\hline & & $\begin{array}{l}\text { DNA methylation } \\
\text { (DAVID) }\end{array}$ & $6.4 \times 10^{-7}$ & $\begin{array}{l}\text { ARID3B, DNMT1, ELAVL1, GNGT2, KANK2, KLF1, LASP1 (P), RAB11B, } \\
\text { RAB3D, SETDB1, SNF8, SRCIN1, SMARCE1, WIPF2, ADGRL1, C17orf96, } \\
\text { CARM1, CCDC117, CPLX3 (P), DIDO1, DOCK6, EEF1A2, HNRNPM, } \\
\text { HIST1H1B, } \\
\text { HIST1H2AI, HIST1H2AI, } \\
\text { HIST1H2AK, HIST1H2AL, } \\
\text { HIST1H2AM, HIST1H2BL, } \\
\text { HIST1H2BM, HIST1H2BN, } \\
\text { HIST1H2BO, HIST1H3H, } \\
\text { HIST1H3I, HIST1H3J, } \\
\text { HIST1H4J, HIST1H4K, } \\
\text { HIST1H4L, HIST2H2AA3, } \\
\text { HIST2H2AA4, HIST2H2AB, HIST2H2AC, HIST2H2BC, } \\
\text { HIST2H2BE, HIST2H3A, } \\
\text { HIST2H3C, HIST2H4A, } \\
\text { HIST2H4B, ILF3, } \\
\text { MED15 (S,P), MRPL4, MYH11, MYL4, NFIX, NCOA3, PALM3, RPRD2, } \\
\text { SEMA7A, ZNF687 }\end{array}$ \\
\hline
\end{tabular}




\begin{tabular}{|c|c|c|c|c|}
\hline Community & $\begin{array}{l}\text { Total number of } \\
\text { nodes/length }(\mathrm{Mb}) \text { in } \\
\text { (sub)community }\end{array}$ & Enrichment in GO term ${ }^{\dagger}$ & p-value & List of genes \\
\hline \multirow[b]{2}{*}{2} & \multirow[b]{2}{*}{$110 / 28.6$} & $\begin{array}{l}\text { DNA demethylation } \\
\text { (GOR) }\end{array}$ & $3.47 \times 10^{-2}$ & $\begin{array}{l}\text { APOBEC } 3 D, A P O B E C 3 A \\
A P O B E C 3 B, A P O B E C 3 C \\
A P O B E C 3 F, A P O B E C 3 H \\
A P O B E C 3 G\end{array}$ \\
\hline & & histone protein class & $1.2 \times 10^{-7}$ & 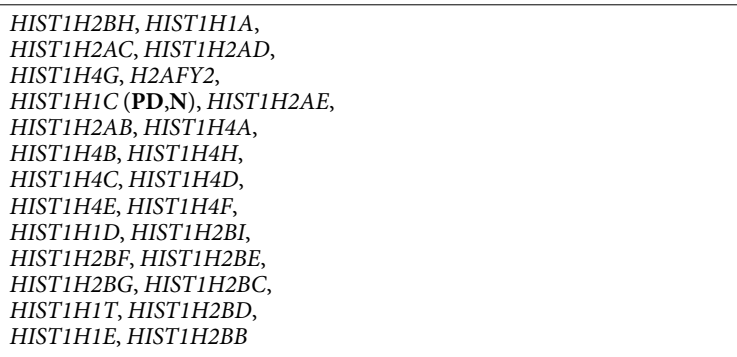 \\
\hline 3 & $154 / 45.5$ & $\begin{array}{l}\text { lipase protein class } \\
(\text { GOR })\end{array}$ & $2.6 \times 10^{-2}$ & $\begin{array}{l}\text { PLD6, PLA2G4E, CES3, LIPC (AD,N,P), CES2, PLA2G1B (S,P), } \\
\text { PLA2G4D (P), LCAT }(\mathbf{N}, \mathbf{P}), \text { CES4A, PLA2G15, PLA2G4B, PLCB2 (S,N,P), } \\
\text { PLA2G4F }\end{array}$ \\
\hline \multirow[t]{2}{*}{4} & \multirow[t]{2}{*}{$164 / 135.1$} & $\begin{array}{l}\text { flavonoid } \\
\text { glucuronidation }\end{array}$ & $\mathrm{FDR}=4.18 \times 10^{-2}$ & $\begin{array}{l}\text { UGT1A3, UGT1A4, UGT1A1, UGT1A5, } \\
\text { UGT1A7, UGT1A10, } \\
\text { UGT1A8, UGT1A6, } \\
\text { UGT1A9 }\end{array}$ \\
\hline & & $\begin{array}{l}\text { vasopressin synthesis } \\
\text { pathway (GOR) }\end{array}$ & $2.25 \times 10^{-3}$ & $\begin{array}{l}\text { NEU1, SEC. } 11 \text { A, SPCS1, } \\
\text { NEU2 }\end{array}$ \\
\hline 5.3 & $11 / 5.3$ & Olfactory receptor & $1.2 \times 10^{-14}$ & $\begin{array}{l}\text { OR10G4, OR10G7, OR10G8, OR10G9, } \\
\text { OR10S1, OR4D5, OR6M1, } \\
\text { OR6T1, OR6X1, OR8A1, } \\
\text { OR8B12, OR8B2, OR8B3, } \\
\text { OR8B4, OR8B8, OR8D1, } \\
\text { OR8D2, OR8D4, OR8G1, } \\
\text { OR8G2, OR8G5 }\end{array}$ \\
\hline \multirow[t]{2}{*}{21} & \multirow[t]{2}{*}{$6 / 5.9$} & $\begin{array}{l}\text { regulation of immune } \\
\text { response }\end{array}$ & $1.62 \times 10^{-2}$ & $\begin{array}{l}\text { CD34, CR1L, } \\
\text { IL10(AD,PD,S,N,P), } \\
\text { MAPKAPK2, CR1, C4BPB, } \\
\text { CD46, CD55, PIGR, } \\
\text { CR2 (PD,N), C4BPA }\end{array}$ \\
\hline & & $\begin{array}{l}\text { regulation of complement } \\
\text { cascade }\end{array}$ & $8.3 \times 10^{-5}$ & $\begin{array}{l}C R 1, C 4 B P B, C D 46, C D 55, \\
C R 2(\mathbf{P D}, \mathbf{N}), C 4 B P A\end{array}$ \\
\hline
\end{tabular}

Table 1. Number of nodes in the selected communities/subcommunities and list of genes enriched in GO terms, identified by Gene Ontology Resource (GOR) and DAVID. Genes present in PGC GWAS dataset are shown in bold. ${ }^{\dagger}$ Only a fraction of GO terms is listed; ${ }^{\ddagger}$ all p-values are corrected for multiple testing: Bonferroni correction in Gene Ontology Resource and Benjamini in DAVID; ${ }^{\S} \mathbf{A D}, \mathbf{P D}, \mathbf{S}, \mathbf{N}$, or $\mathbf{P}$, next to the specific gene, indicates its association with Alzheimer's, Parkinson's or schizophrenia disease, neurological or psychological disease class, respectively, as identified by DAVID software.

degree (number of interactions with other regions) of their corresponding nodes. The latter region together with all connected regions harbours 7200 genes (Supplementary Table S4) enriched in several GO terms. For example, 580 genes were enriched in the GO cellular component term "neuron part". Among these genes there were 21 genes (e.g. CHRNA2, CHRNA5, CACNA1C and CACNA1D) from the SCZ high-confidence gene list ${ }^{19}$ and 26 genes (e.g. CHRNA3, CLU, GRIN2A, MAPK8IP1 and PARL7) from the SCZ risk gene list ${ }^{19}$. One of the novel genes found in this group is flotillin 1 gene, FLOT1, located on chromosome 6 . This gene is highly expressed in the brain and harbours 15 SNPs with one of them, rs1059612, reaching genome wide significance level $\left(\mathrm{p}=1.03 \times 10^{-}\right.$ $\left.{ }^{8}\right)$ and another one, rs 1064627, having p-value just below significance level $\left(p=8.39 \times 10^{-7}\right)$. Recent studies ${ }^{18}$ involving larger datasets did not show a strengthened association of these SNPs with SCZ. Nevertheless, the STRING interaction network provided by GeneCard (https://www.genecards.org/), shows protein-protein interactions of FLOT1 protein with LRFN3 and CORO1C proteins. Our analysis shows that these genes also occur within close proximity to the FLOT1 gene, i.e. all three of them interact with chrX:20,500,001-21,900,000 region and are present in the set of 580 genes enriched in "neuron part" GO term although none of them are present in SCZ high-confidence or risk gene lists ${ }^{19}$. The leucine rich repeat neuronal 3 gene, LRFN3, although not present in the expanded PGC dataset and the recent GWAS datasets ${ }^{18}$, has been found to be associated with schizophrenia and severe progressive autism by other GWAS (https://www.genecards.org/). One can speculate that existence of protein-protein interactions, close proximity of these genes within the nucleus and shared biological function, makes the FLOT1 gene (as well as other genes from this set of 580 genes) a potential candidate, linked to schizophrenia. SNPs residing within these genes, not necessarily meeting genome-wide significance threshold, may still cause the phenotype, but either collectively, i.e. together with other SNPs residing within these co-located genes, or selectively when SNPs in different functionally-related genes could show significant association with the phenotype in a smaller subgroup, e.g. in a subgroup of patients with both schizophrenia and severe progressive autism. Note that the absence of CAV1, SCC6A3 and SVIL genes, which exhibit protein-protein interaction with 

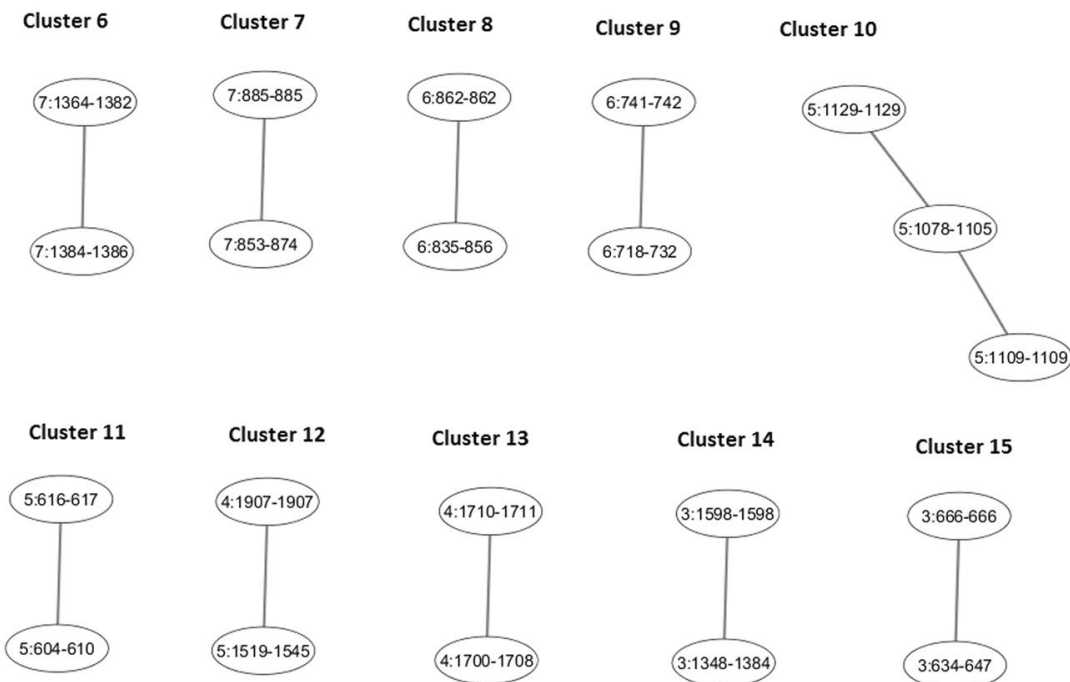

Cluster 15

Cluster 16

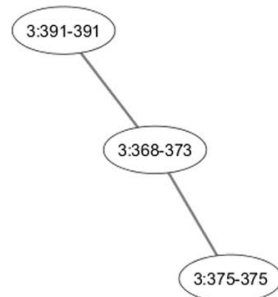

Cluster 17

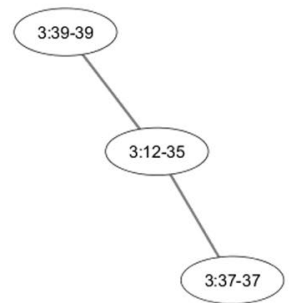

Cluster 18

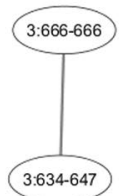

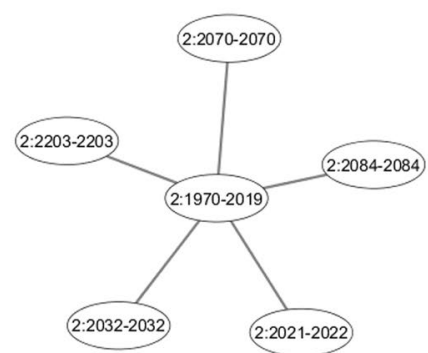

Cluster 19
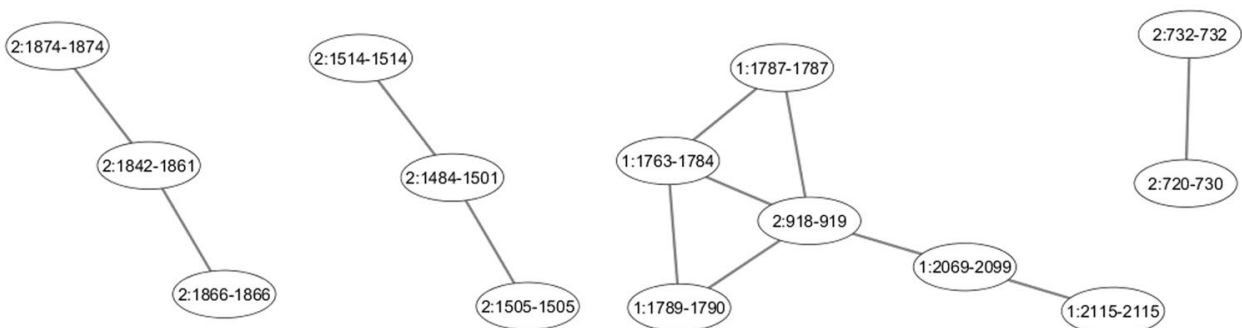

Figure 1. Small $(12,21)$ and isolated $(6-11,13-20,22)$ clusters identified in the extended network of interaction.

FLOT1, from the resulting dataset of genes is likely to be due to our stringent selection of inter-chromosomal interactions by considering only $0.05 \%$ of the strongest interactions, recorded in $\mathrm{Hi}-\mathrm{C}$ datasets.

Disconnected subnetworks. The occurrence of SNPs in genes residing within isolated subnetworks could indicate their association with a specific manifestation of schizophrenia in groups of patients stratified by e.g. schizophrenia sub-phenotypes. Although none of the SNPs residing within 31 SCZ-associated genes comprising 13 disconnected subnetworks reaches the genome-wide significance level in the expanded PGC dataset used, several later studies showed their significant association with SCZ. Fromer et al. ${ }^{20}$ have identified five single-gene loci with three of them - CNTN4, CLCN3 and SNAP91 - residing respectively within subnetworks 17, 13 and 8 (Fig. 1, Supplementary Fig. S2 and Table S5) with the remaining two genes, FURIN and TSNARE1, residing within cluster 4 (see Fig. 3 and explanation below). Fromer et al. ${ }^{20}$ have shown that some SNPs in these genes could alter the expression of these genes in patients with schizophrenia. All these genes were later found to harbour significant $\mathrm{SNPs}^{18}$. In addition, our analysis of the extended network shows that the promoter of the CNTN4 gene (cluster 17 in Fig. 1 and Supplementary Fig. S2), which is a member of the contactin family of immunoglobulins and plays a role in the development of nervous system, has strong interactions with the promoter regions of the LRRN1 gene (node labelled 3:39-39 in subnetwork 17); nine SNPs residing within this promoter region could also contribute to the change in expression of the CNTN4 gene. The LRRN1 gene itself, which was not in our expanded 


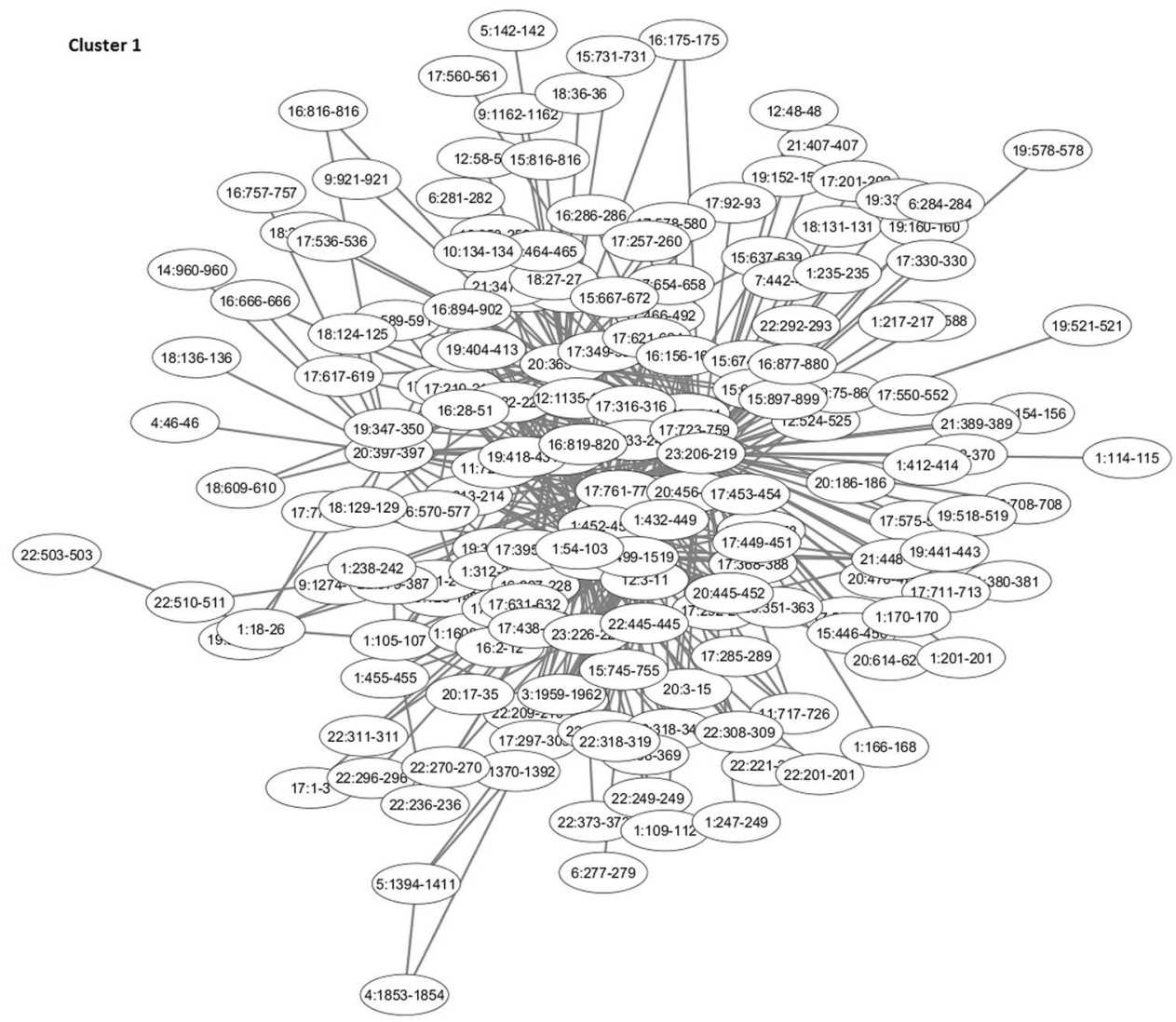

Cluster 2

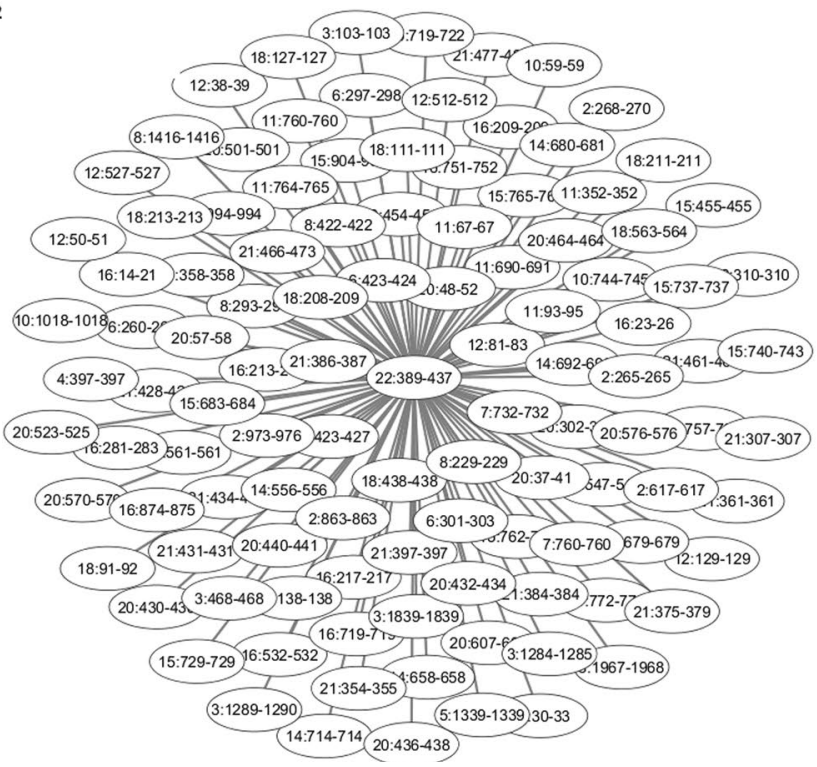

Figure 2. Clusters 1 and 2 identified in the extended network of interaction.

PGC dataset and is not present in the recent set SCZ-associated genes ${ }^{18}$, was reported to have an altered RNA expression in blood of patients with Alzheimer's disease ${ }^{21}$. Another gene, the synaptosome associated protein 91 SNAP91, was present in the expanded PGC dataset harbouring 75 SNPs (albeit with p-values $\geq 7.86 \times 10^{-4}$ ). It was later shown ${ }^{18}$ that it harbours a SNP rs217331 $\left(\mathrm{p}=1.17 \times 10^{-12}\right)$. The promoter of this gene has a strong intra-chromosomal interaction with the NT5E gene promoter region not previously found to associate with SCZ. Garcia-Esparcia et al. ${ }^{22}$ found that the $5^{\prime}$-nucleotidase gene, NT5E, is involved in purine metabolism and deregulated in Parkinson's disease brains. These two examples demonstrate that SNPs in these co-located genes could be important in smaller subgroups of SCZ patients also suffering either from Alzheimer's or Parkinson's disease. 
One can also speculate about a possible existence of a shared genetic mechanism underlying several psychological and neurodegenerative disorders.

Interestingly, some of the SNPs found in genes residing within the isolated subnetworks are associated with specific manifestation of schizophrenia and, possibly, of a different sub-phenotype. For example, the MAN2A1 gene residing within an isolated cluster 10 (Fig. 1 and Supplementary Fig. S2) was previously found to associate with SCZ $\left(p=3.05 \times 10^{-08}\right)$ but was discarded from the list of SCZ-associated genes based on the results of meta-analysis (see ref. ${ }^{18}$ and Supplementary Table 5 therein). This particular gene was found to associate with eye movement dysfunction in schizophrenia ${ }^{23}$ and may not show significant association with SCZ when patients are not stratified by sub-phenotypes; the occurrence of this gene within an isolated subnetwork may indicate its association with specific manifestation of schizophrenia.

Clusters. Nine genes in cluster 4 (Fig. 3) were found to be enriched in the "flavonoid glucuronidation" GO term $\left(F D R=4.18 \times 10^{-2}\right)$. None of these genes were present in the expanded PGC dataset but were reported elsewhere as associated with SCZ. Among them are the UGT1A1 and UGT1A4 genes (UDP glucuronosyltransferase family 1 member A1 and A4), which harbour 20 and 58 SNPs, respectively, with p-values above $4.1 \times 10^{-2}$. Erickson-Ridout et al. ${ }^{24}$ have shown that two polymorphisms in these genes significantly alter glucuronidation of the antipsychotic drug Clozapine, used for treating refractory schizophrenia. These polymorphisms were found to be important in determining inter-individual differences in drug metabolism in vivo. Other newly found genes within this cluster, also involved in glucuronidation and co-located with the two known genes, could provide new screening targets and help to determine the right dosage, patient response and side effects to either Clozapine or other drugs being developed for treating schizophrenia.

In total 716 genes, residing within cluster 2 (Fig. 2) and including the histone cluster gene family (prefix HIST1) genes, were enriched in the "histone" protein class and "DNA demethylation" (Table 1). Four of these genes (HIST1H2BD, HIST1H2BC, HIST1H2BH and HIST1H2BG) form a part of the major histocompatibility complex (xMHC) region on chromosome 6. Several GWAS have shown its strong association with schizophrenia (see, for example, ref. ${ }^{25}$ ). Using whole genome gene expression profiling, Sanders et al. ${ }^{26}$ identified a set of 95 differentially expressed transcripts enriched in immune-related genes, listed above. Other genes from the extended xMHC, e.g. HIST1H4C, BTN3A3 and ZNRD1 (also in cluster 2), were also found as potential schizophrenia genes $^{27}$. The latter harbour a SNP with $\mathrm{p}$-value meeting the genome-wide significance level $\left(\mathrm{p}=8.80 \times 10^{-9}\right)$. Recent results of meta-analyses of CLOZUK and PGC GWAS data ${ }^{18}$ showed the improved significance of SNPs in this region ( 12997 SNPs in total, $p=4.32 \times 10^{-44}$ for the index SNP rs1233578). Note that the primary function of the family of histone genes is to facilitate the organisation of DNA on chromatin. Hence, SNPs affecting these genes may disrupt the local 3D architecture of the human genome either collectively, when several genes are disrupted in the same patient, or individually if SNPs show high association with a given phenotype, thus perhaps resulting in the SCZ phenotype as reported in ref. ${ }^{27}$. One can speculate that other genes from the histone cluster gene family found in this community (see Table 1) could also contribute to schizophrenia risk.

In addition, two out of 511 SNPs residing on chr6:25,900,001-26,600,000 region in cluster 2, rs3857547 $\left(\right.$ chr6:26157762; $\left.\mathrm{p}=1.46 \times 10^{-9}\right)$ and rs7749823 $\left(\mathrm{chr6:26158079;} \mathrm{p}=6.01 \times 10^{-9}\right)$, reach genome-wide significance level of association with schizophrenia. ENCODE data indicates that these SNPs occur within an enhancer/ promoter, targeting several genes including HIST1H2BD, HIST1H1E, BTN2A2, BTN2A1, BTN2A3P, BTN3A1, $B T N 3 A 2$ and $B T N 3 A 3$ also residing within cluster 2 and therefore located in close proximity to each other within the cell nucleus. It is possible that this enhancer/promoter, found in several cell lines and tissues including H1 neuronal progenitor and GM12878, could target other remotely located genes residing within this cluster.

DNA methylation has been implicated in the etiopathology of various complex disorders. Several studies have examined DNA methylation changes in schizophrenia. For example, rs2114724 (not present in the expanded PGC dataset) and rs2228611 SNPs in the DNA methyltransferase 1 gene, DNMT1, increase the risk of developing schizophrenia in males or are associated with early onset of schizophrenia and with family history in South Indian population $^{28}$. Although cluster 2 was not found to be enriched in "DNA methylation" GO term, six genes of the butyrophilin family (BTN3A2, BTN2A3P, BTN2A1, BTN3A3, BTN3A1, BTN2A2) were found to reside within this cluster. Correlation between DNA methylation and gene expression in these four genes, including the butyrophilin gene $B T N 3 A 3$, found in patients with SCZ and bipolar disorder was reported in ref. ${ }^{29}$. One can speculate that DNA methylation of the butyrophilin genes correlates with their expression in a way, similar to the BTN3A3 gene $^{29}$; their methylation patterns could be novel candidate factors conferring risk to schizophrenia in patients either with bipolar disorder or an early onset of schizophrenia.

Six genes - CR1, C4BPB, CD46, CD55, CR2, C4BPA - in cluster 21 were enriched in the "regulation of complement cascade" GO term. Håvik et al. ${ }^{30}$ demonstrated the significant role of complement control-related genes in the etiology of schizophrenia and support disease mechanisms that involve the activity of immunity-related pathways in the brain. Several common SNPs in the complement control-related gene CSMD2 and its homolog CSMD1 and in the complement surface receptor CD46 residing in cluster 21 have exhibited an association with SCZ across three large samples comprising 1133 patients and 2444 healthy controls ${ }^{30}$, although the p-values obtained by recent meta-analysis ${ }^{18}$ no longer show significant association. According to the DAVID database, the complement C3d receptor 2 gene, CR2, has been also found in association with Parkinson's and other neurological disorders. Further analysis of other complement control-related genes found within this cluster is required to prove their association with SCZ. Interestingly, the association of the major histocompatibility complex locus with SCZ was partly explained by structurally diverse alleles of the complement component 4 (C4) genes ${ }^{31}$. As a result, varying levels of $\mathrm{C} 4 \mathrm{~A}$ and $\mathrm{C} 4 \mathrm{~B}$ expression in the brain are generated, implicating excessive complement activity in the development of schizophrenia that leads to the reduced numbers of synapses in the brains of individuals with schizophrenia. 

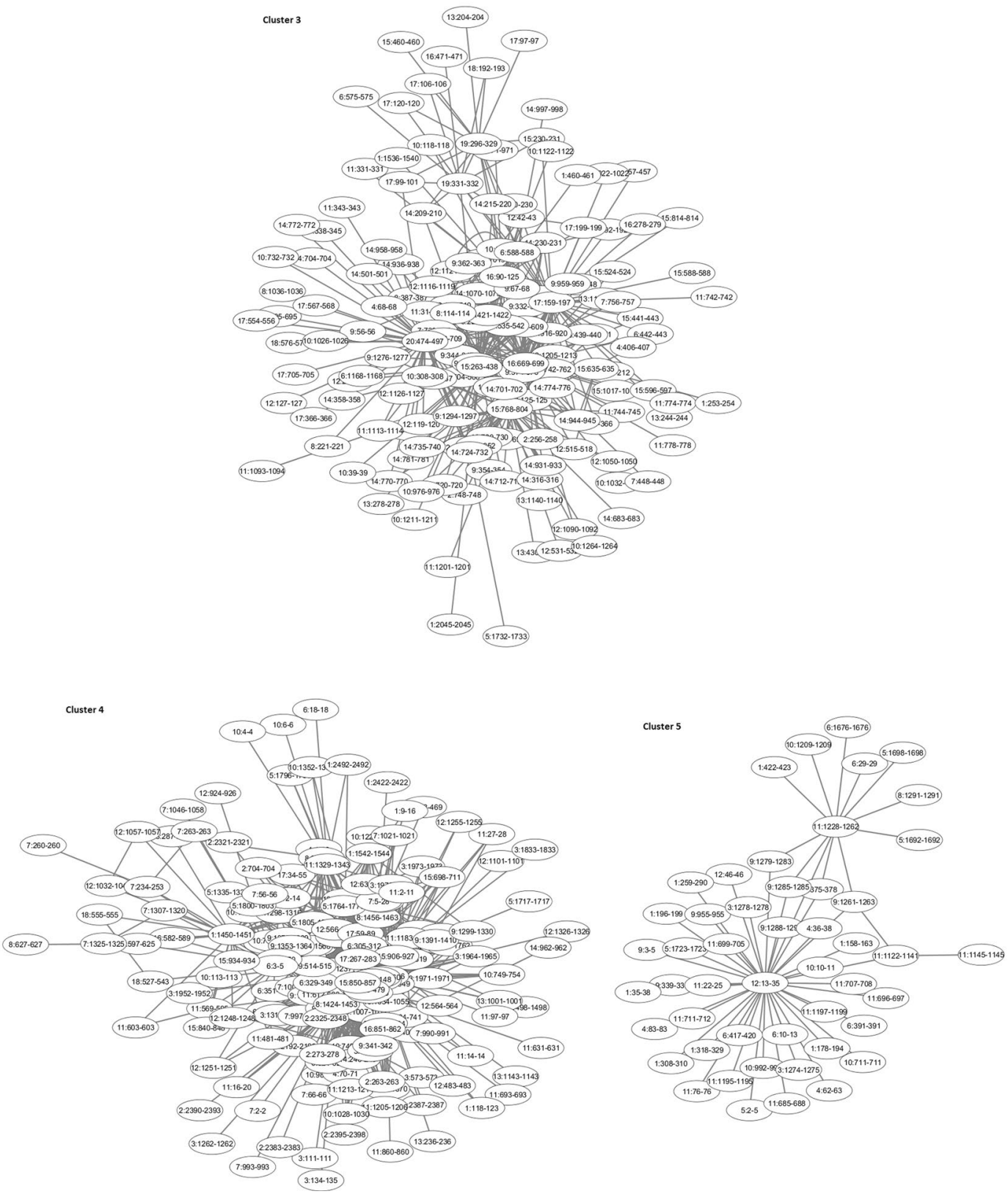

Figure 3. Clusters 3-5 identified in the extended network of interaction.

Three genes in cluster 1.2 - HN1, MMP24, LRP5 - were enriched in the "vasopressin synthesis" pathway (Table 1). Vasopressin and oxytocin were found to be associated with cognitive and clinical symptoms severity in midlife women with chronic schizophrenia ${ }^{32}$. The DAVID database also shows an association of the MMP24 gene with Parkinson's disease. These observations show that although SNPs in these three genes do not exhibit genome-wide significance, they may still play an important role, but in a smaller well-defined subgroup of patients. In the same vein, down-regulation of the olfactory receptors, co-located in cluster 5.3, in the dorsolateral prefrontal cortex in patients with chronic schizophrenia was noted by Ansoleaga et al. ${ }^{33}$.

Cluster 1.3 was enriched in the "DNA methylation" GO term (DAVID, $\mathrm{p}=6.4 \times 10^{-7}$ after Benjamini correction), "histone" protein class (GOR, $\mathrm{p}=6.32 \times 10^{-8}$ ) and "histone fold" (DAVID, $\mathrm{p}=4.6 \times 10^{-12}$ ). The latter set of 25 genes is a subset of 60 genes enriched in the "DNA methylation" GO term (Table 1). Eight out of 122

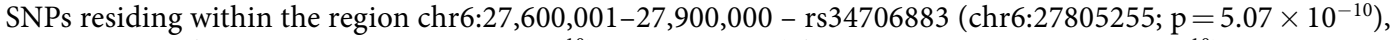
rs13212651 (chr6:27806985; $\left.\mathrm{p}=6.2 \times 10^{-10}\right), \mathrm{rs} 17693963\left(\operatorname{chr} 6: 27710165 ; \mathrm{p}=1.56 \times 10^{-10}\right), \mathrm{rs} 13194781$ $\left(\mathrm{chr6:27815639;} \mathrm{p}=5.45 \times 10^{-10}\right), \mathrm{rs} 13199772\left(\mathrm{chr6:27834085} ; \mathrm{p}=7.05 \times 10^{-10}\right)$, rs $17749927(\mathrm{chr6:27669976}$; $\left.\mathrm{p}=1.01 \times 10^{-9}\right)$, rs $17750424\left(\mathrm{chr6}: 27701122 ; \mathrm{p}=1.49 \times 10^{-9}\right)$ and $\mathrm{rs} 13218875\left(\mathrm{chr} 6: 27884012 ; \mathrm{p}=1.02 \times 10^{-8}\right)$ - reached genome-wide significance level for association meaning that each of these SNPs may cause the observed phenotype, but in different patients. Moreover, according to the ENCODE data the first two SNPs that reside within enhancer/promoter regions target various genes in the histocompatibility complex region as 
well as remotely located glutathione peroxidase 5 and 6 genes, GPX5 (chr6:28,493,789-28,502,728) and GPX6 chr6:28,471,073-28,483,570), known to protect cells and enzymes from oxidative damage. These two genes were also found to associate with $\mathrm{SCZ}^{34}$. Interestingly, the region harbouring these SNPs may form looping interactions with the promoters of the GPX5 and GPX6 genes; a strong intra-chromosomal interaction is recorded between these regions in Capture Hi-C data. Expression of other genes residing within this community could be also influenced by SNPs found on chr6:27,600,001-27,900,000.

Five genes from the haemoglobin subunit gene family ( $H B M, H B A 2 / H B A 1, H B Z, H B Q 1)$ located on chr16:100,001-900,000 in cluster 1.1 were enriched in the "oxygen transport" GO term. The role of genes of the haemoglobin family is to transport oxygen throughout the body via red blood cells; alterations in these genes could prohibit a sufficient amount of oxygen from entering the brain, which is a cause known to increase the risk of SCZ in adults ${ }^{35}$. The emerging role of haemoglobins, as a potentially novel mechanism underlying mental disorders, was discussed by Altinoz \& Ince ${ }^{36}$.

Twenty-four genes residing within cluster 1 were enriched in the "Alzheimer disease (AD)-presenilin pathway". Several genes from this cluster were also implicated in neurodevelopmental disorders and schizophrenia. Prenatal viral-like immune activation in a mouse model displayed long-term epigenetic modification in Wnt-signalling genes Wnt 3 and $W n t 8 a$ in adult offspring ${ }^{37}$, prompting a plausible explanation for the disruption of prefrontal gene transcription and behavioural functions in patients with prenatal infectious histories. Passos Gregorio et al ${ }^{38}$ studied the link of non-synonymous variants in five genes of the Notch-signalling pathway NOTCH2, NOTCH3, JAGGED2, ASCL1 and NUMBL - to schizophrenia in two independent and unrelated populations. The authors found that only SNPs in the NUMBL gene, which also resides within cluster 1, have an association with schizophrenia. The ASCL1-harbouring locus was also one of the new loci, identified in ref. ${ }^{18}$. Gao et al. ${ }^{39}$ reported a specific abnormality in peripheral expression of the matrix metalloproteinase 9 gene, $M M P 9$, and its methylation, indicating a potentially different pathological mechanism underlying a specific subgroup of schizophrenia, which includes deficit schizophrenia patients. In turn, gene expression profiling of the dorsolateral and medial orbitofrontal cortex in schizophrenia patients ${ }^{40}$ identified seven novel genes, including KAT7 and EVI2A, and previously reported gene TARDBP (all residing within cluster 1) that are differentially expressed in several brain regions, including prefrontal cortical regions, in patients with SCZ. Fazzari et al.$^{41}$ have demonstrated the physiological role of Aph1b- $\gamma$-secretase $(A P H 1 B)$ in brain and its relevance to schizophrenia. We speculate that enrichment of SCZ genes identified via network analysis, which takes into account the proximity of genes within the cell nucleus, in "Alzheimer disease-presenilin pathway" GO term may imply that both AD and schizophrenia share a common genetic risk at least for some specific phenotypes, e.g. deficit schizophrenia as reported in ref. ${ }^{39}$.

A finer structure of intra-chromosomal interactions was explored by analysing interactions within extended gene regions. For example, the EGR region on chr17:400,001-32,00,000, residing within community 4.1, harbours 58 genes, including four genes, TSR1, SGSM2, SMG6 and SRR, that were present in the expanded PGC dataset. The $S R R$ gene, which encodes an enzyme that synthesizes D-serine from L-serine, was involved in a co-expression module associated with schizophrenia disease status ${ }^{42}$. The authors have also shown that a SNP, rs 16952025 ( $\mathrm{p}=0.47$ in the expanded PGC dataset), residing $\sim 90 \mathrm{~K}$ upstream of the $S R R$ gene within an intron of the neighbouring SMG6 gene, was significantly associated with the expression level of the SRR gene. We found that the region harbouring this SNP has a strong intra-chromosomal interaction with the SRR gene promoter; it is plausible that this SNP occurs within an enhancer that controls the SRR gene expression via chromatin looping interaction. Although no association with the expression of the SMG6 gene itself was found ${ }^{42}$, the association study performed by Tabarés-Seisdedos et al. ${ }^{43}$ on schizophrenia and bipolar patients from a Spanish isolated population found an association between structural variant in the SMG6 gene and prefrontal cognitive deficit in patients with SCZ and bipolar disorder. In the subsequent studies ${ }^{18}$, the locus harbouring the TSR1, SGSM2, $S M G 6$ and SRR genes was also found to be significantly associated with SCZ. Two genes, SMG6 and SRR, were also found as genes strongly associated with $\mathrm{SCZ}^{19}$.

Two nodes in a two-node isolated subnetwork 12, connecting regions chr5:151,800,001-154,500,000 and chr4:190,600,001-190,700,000, was looked at in greater details. The region on chromosome 5 harbours 18 genes and 1264 SNPs (the smallest $\mathrm{p}=7.23 \times 10^{-6}$ for rs12522297), including the GRIA1 and GALNT10 from the expanded PGC dataset harbouring 221 and 181 SNPs with p-values above $2.75 \times 10^{-4}$, respectively. No genes were found in the region on chromosome 4 (UCSC Genome Browser, GRCh37/hg19; http://genome-euro.ucsc.edu). Fourteen SNPs were found in this region; none of them have reached genome-wide significance level $\left(\mathrm{p} \geq 5.36 \times 10^{-4}\right)$. Interestingly, this region harbours 161 transcription factor binding sites (TFBS), identified by ChIP-Seq technology. It is commonly accepted that transcription factors that bind to TFBS either activate or repress the transcription of the target gene(s) that usually reside in the close proximity of the TFBS. With the new patches, available for hg19 assembly, only one RNA gene - long intergenic non-protein coding RNA 1262 (LINC01262) - was found within the adjacent $100 \mathrm{~Kb}$ region upstream of the TFBS-enriched regions. Diseases associated with LINC01262 include late-onset of Parkinson's disease (https://www.genecards.org/). Several genes/pseudogenes (DUX8L8, DUX4L6, DUX4, DUX4L4, DUX4L5, TUBB4Q and FRG1/2) were found more than 200Kb downstream of the TFBS-enriched regions. The contraction of the macro-satellite repeats in DUX-pseudogenes and centromeric deletion involving the FRG1 gene were reported as a cause of muscular dystrophy in general and facioscapulohumeral muscular dystrophy in particular. No disorders were recorded in GeneCards for tubulin beta 7 pseudogene TUBB7P and the LINC01596 RNA gene residing more than $200 \mathrm{~Kb}$ downstream of the TFBS-enriched regions. One can speculate that some of the target genes may reside within strongly interacting region, chr5:151,800,001-154,500,000, and TFBS influence the expression of one or several of the 19 genes residing within this region.

In conclusion, this study provided the evidence for using co-location of genes/genomic regions governed by the $3 \mathrm{D}$ architecture of the human genome for predicting genes and genomic regions linked to a specific phenotype. Using our network approach, several novel candidate regions were found to harbour genes with prior 
reported or recently confirmed associations with SCZ, acting as a proof of concept. The use of networks of intraand inter-chromosomal interactions for relevant cell lines or tissues, coupled with the information on the enrichment of respectively coding and non-coding regions in various schizophrenia-related GO terms and functional elements pave the way for understanding the contribution of seemingly remote SNPs/genes to polygenic disorders, such as SCZ, and provide a plausible biological explanation for their cooperation. This approach therefore promises to be of potential utility in identifying novel genes/genomic regions linked to other polygenic disorders and the means of aggregating genes/SNPs according to their co-location and shared biological function for further investigation, e.g. by using polygenic scores.

\section{Materials and Methods}

Dataset of SNPs used in this study. In this study we further expanded a dataset of SNPs downloaded from the Psychiatric Genomics Consortium website https://www.med.unc.edu/pgc/results-and-downloads/downloads (permission was obtained in 2012) by SNPs found via review of literature and the use of various bioinformatics resources. The resulting dataset comprised 1,252,901 SNPs spanning 22 chromosomes. Variants on chromosome $\mathrm{X}$ were not considered. Each SNP has an accompanying summary statistic that includes a p-value, showing the strength of association with SCZ. The p-values lie within the range $4.3 \times 10^{-11}<\mathrm{p}<0.998$ with only 136 SNPs reaching the genome-wide significance threshold of $\mathrm{p}<5 \times 10^{-8}$. A total of 346 genes with putative association with SCZ, identified by the PGC ${ }^{6,7}$ and other studies, were used. We refer to these datasets of SNPs and genes as expanded PGC datasets. When required, genomic positions of SNPs and genes were 'lifted over' to the hg19 assembly using the Lift Genome Annotation program available at https://genome.ucsc.edu/cgi-bin/hgLiftOver.

Inter- and intra-chromosomal interaction data. At the time when this study was initiated, inter- and intra-chromosomal interaction data were available for nine human cell lines, including karyotypically normal B-lymphoblastoids (GM12878), lung fibroblasts (IMR90) and mammary epithelial (HMEC). Interaction frequencies between $100 \mathrm{~Kb}$ regions (bins) and bins ranging from $1 \mathrm{~Kb}$ to $100 \mathrm{~Kb}$ for respectively inter- and intra-chromosomal contacts generated by in situ Hi-C method (FDR $<10 \%)^{10}$ were downloaded from http:// www.ncbi.nlm.nih.gov/geo/ (accession number GSE63525). Henceforth, we refer to this data as in situ Hi-C data. Normalisation coefficients, accounting for biases introduced by experimental procedure and intrinsic properties of the human genome, were also available alongside raw counts. In this study we used inter-chromosomal contact frequencies, normalised using inter-chromosomal Knight and Ruiz normalisation procedure. In addition, a dataset of intra-chromosomal interactions between approximately 22,000 gene promoter regions and their potential enhancers, and between gene promoters of various genes obtained using the Capture Hi-C method ${ }^{11}$, were downloaded from http://www.ebi.ac.uk/arrayexpress/experiments/ (accession number E-MTAB-2323). Only significantly interacting regions filtered from a background noise using a one-sided cumulative binomial test and Benjamini-Hochberg correction for multiple testing with FDR $<0.05$ were recorded in these datasets ${ }^{11}$.

Brain expression quantitative trait loci data. The dataset of brain-related expression quantitative trait loci (eQTL) was downloaded from the Brain Expression Quantitative Trait Loci (eQTL) Almanac (Braineac) ${ }^{12}$. This dataset contains SNPs affecting the expression of target genes in ten distinct brain regions: the cerebellar cortex (CRBL), frontal cortex (FCTX), hippocampus (HIPP), inferior olivary nucleus (sub-dissected from the medulla; MEDU), occipital cortex (OCTX), putamen (PUTM), substantia nigra (SNIG), temporal cortex (TCTX), thalamus (THAL) and intralobular white matter (WHMT). Each SNP in this dataset is paired with a target gene in cis or in trans and an associated p-value. Small p-values correspond to a more profound target gene expression change in a given brain tissue and therefore indicate a stronger affinity to being associated with psychotic disorders. An expression averaged across all ten regions (aveALL) is also available within Braineac ${ }^{12}$.

Selecting cell line to best emulate 3D interactions in brain tissues. To determine which cell line most closely resembled interactions in brain tissues, brain eQTL data ${ }^{12}$ was used. To align the in situ intra-chromosomal Hi-C data ${ }^{10}$ with the eQTL data, each cis-eQTL pair positions of SNPs and their targets were uniquely binned into regions in multiples of $10 \mathrm{~Kb}, 100 \mathrm{~Kb}$ and $1 \mathrm{Mb}$ if the distances between SNPs and their targets were $<100 \mathrm{~Kb},<1 \mathrm{Mb}$ and $>1 \mathrm{Mb}$, respectively. All pairs for which both a SNP and its target occurred within the same $10 \mathrm{~Kb}$ bin were deleted. If several SNPs residing in the same bin were targeting the same gene, only one cis-eQTL pair with the lowest p-value, indicating significant change in expression, was retained. For each of the three cell lines (GM12878, IMR90 and HMEC), the number of cis-eQTL pairs that exhibit strong interactions (or, in other words, are nearby in 3D) was counted. The cell line exhibiting the highest enrichment of intra-chromosomal interactions in brain-related eQTL was used in subsequent analyses.

Extended gene regions (EGRs). Capture Hi-C data ${ }^{11}$ was used to identify potential regulatory regions by considering all intra-chromosomal regions strongly interacting with promoters of each of the 346 SCZ-associated genes. A promoter-other_significant_interactions dataset was used to identify potential enhancers, whilst promotor regions of other genes interacting with a given gene promoter and potentially influencing its expression were identified from promoter-promoter_significant_interactions dataset. Promoter and enhancer boundaries were calculated from start and end positions of each interacting pair of fragments; a genomic boundary was then drawn encompassing all overlapping fragments that belong to the same gene promoter or enhancer. Positions of genes and their associated regulatory regions were binned into regions in multiples of $100 \mathrm{~Kb}$. In cases where several $100 \mathrm{~Kb}$ regions harbouring genes and/or their regulatory elements were adjacent to each other, they have been amalgamated into a longer extended gene region (EGR), sometimes spanning several $100 \mathrm{~Kb}$ bins and comprising several genes. The intra-chromosomal interactions between all non-overlapping and non-adjacent EGRs, promoter- and enhancer-harbouring regions were preserved (see Supplementary Fig. S3). 
Network approach to identify schizophrenia candidate regions. First, for a set of non-overlapping extended gene regions of $346 \mathrm{SCZ}$-associated genes and regions harbouring promoters and enhancers (as defined above), a network was created where nodes representing these regions were connected by edges if a strong inter-chromosomal interaction between at least one pair of $100 \mathrm{~Kb}$ fragments constituting these regions was recorded in Capture Hi-C data. All intra-chromosomal interactions identified earlier (see Extended gene regions section) were added to the network. Normalised inter-chromosomal contact frequencies between $100 \mathrm{~Kb}$ fragments exceeding a predefined threshold were used to measure the strength of the interactions, which were inversely proportional to their closeness within the cell nucleus. Thus, the resulting network, henceforth called the original network, comprises nodes corresponding to the extended regions of the $346 \mathrm{SCZ}$-associated genes and/or their regulatory regions and possible interactions between these regions that arise due to their close spatial proximity within the cell nucleus.

Next, new nodes and edges were added to the original network by including all $100 \mathrm{~Kb}$ regions that have strong (above a predefined threshold) inter-chromosomal interactions with the nodes from the original network, or in other words, by adding first nearest neighbours to the nodes of the original network. All adjacent regions have been amalgamated into longer regions, and links between amalgamated nodes were added (or kept) if at least one $100 \mathrm{~Kb}$ fragment from these longer regions interacted with $100 \mathrm{~Kb}$ fragments constituting either another 100 $\mathrm{Kb}$ or an amalgamated region. We refer to this network as the extended network.

To identify important nodes within the network, for each node its degree was calculated as a number of connections a node has. The choice of this specific measure was largely predefined by the way networks were created by considering only first nearest neighbours of the SCZ-associated regions. In addition, the Girvan-Newman fast greedy algorithm ${ }^{44}$ implemented as GLay plugin ${ }^{45}$ (Su et al., 2010) to Cytoscape ${ }^{46}$ was used to detect communities within the resulting network, i.e. groups of nodes/regions that are densely connected to each other within a given community but sparsely connected to nodes in other communities of the network.

Additional bioinformatics resources used to investigate candidate regions. Upon identifying SCZ candidate regions (with some of them being $>100 \mathrm{~Kb}$ long) using the aforementioned network approach, various bioinformatics resources were used to narrow down the length of regions found and to identify novel genes/loci that have the potential to be associated with schizophrenia. After compiling sets of genes residing within nodes/candidate regions, which belong to a certain network community, the enrichment of the resulting sets of genes in various gene ontology $(\mathrm{GO})$ terms have been sought.

Three independent gene ontologies, compiled by the Gene Ontology Consortium ${ }^{14,15}$ and available at http:// www.geneontology.org, together with the Database for Annotation, Visualisation and Integrated Discovery $(\text { DAVID })^{16}$ were used to assess the enrichment of a given set of genes in biological processes, molecular functions and cellular components. In addition, DAVID was used to identify whether any gene from a given set of genes was linked to two disease classes (neurological and physiological) and a certain disease (schizophrenia). Only GO terms with high enrichment scores and low $\mathrm{p}$-values ( $\mathrm{p}<0.05$ after correction for multiple testing) were considered.

\section{Data availability}

List of 346 genes with putative association with SCZ, identified by the $\mathrm{PGC}^{6,7}$ and via an extensive literature search is given in Supplementary Table S1. List of extended genomic regions that do not interact with any other loci is given in Supplementary Table S2. Genes residing within nodes chr22:38,800,001-43,700,000 (degree 328) and chrX:20,500,001-21,900,000 (degree 304) and connected regions are listed in Supplementary Tables S3 and S4, respectively. Complete sets of genes residing within nodes, comprising each community, are given in Supplementary Table S5.

Received: 17 July 2019; Accepted: 13 November 2019;

Published online: 29 November 2019

\section{References}

1. Maurano, M. T. et al. Systematic localization of common disease-associated variation in regulatory DNA. Science 337, 1190-1195 (2012).

2. Bhatia, S. \& Kleinjan, D. A. Disruption of long-range gene regulation in human genetic disease: a kaleidoscope of general principles, diverse mechanisms and unique phenotypic consequences. Hum. Genet. 133, 815-845 (2014).

3. Mumbach, M. R. et al. Enhancer connectome in primary human cells identifies target genes of disease-associated DNA elements. Nat. Genet. 49, 1602-1612 (2017).

4. de Leeuw, C. A., Mooij, J. M., Heskes, T. \& Posthuma, D. MAGMA: Generalized gene-set analysis of GWAS data. PLoS Comput. Biol. 11, e1004219, https://doi.org/10.1371/journal.pcbi.1004219 (2015).

5. International Schizophrenia Consortium, Purcell, S. M. et al. Common polygenic variation contributes to risk of schizophrenia and bipolar disorder. Nature 460, 748-752 (2009).

6. Schizophrenia Psychiatric Genome-Wide Association Study (GWAS) Consortium, Ripke, S. et al. Genome-wide association study identifies five new schizophrenia loci. Nat. Genet. 43, 969-976 (2011).

7. Schizophrenia Working Group of the Psychiatric Genomics Consortium, Ripke, S. et al. Biological insights from 108 schizophreniaassociated genetic loci. Nature 511, 421-427 (2014).

8. Jia, P., Wang, L., Meltzer, H. Y. \& Zhao, Z. Common variants conferring risk of schizophrenia: A pathway analysis of GWAS data. Schizophr. Res. 122, 38-42 (2010).

9. Lieberman-Aiden, E. et al. Comprehensive mapping of long-range interactions reveals folding principles of the human genome. Science 326, 289-293 (2009).

10. Rao, S. S. et al. A 3D map of the human genome at kilobase resolution reveals principles of chromatin looping. Cell 159, 1665-1680 (2014).

11. Mifsud, B. et al. Mapping long-range promoter contacts in human cells with high-resolution capture Hi-C. Nat. Genet. 47, 598-606 (2015). 
12. Ramasamy, A. et al. Genetic variability in the regulation of gene expression in ten regions of the human brain. Nat. Neurosci. 17, 1418-1428 (2014).

13. Estrada, E. The structure of complex networks: Theory and applications. 121-215 (Oxford University Press, 2012).

14. Ashburner, M. et al. Gene ontology: tool for the unification of biology. The Gene Ontology Consortium. Nat. Genet. 25, 25-29 (2000).

15. Mi, H., Muruganujan, A., Casagrande, J. T. \& Thomas, P. D. Large-scale gene function analysis with the PANTHER classification system. Nat. Protoc. 8, 1551-1566 (2013).

16. Huang, D. W., Sherman, B. T. \& Lempicki, R. A. Systematic and integrative analysis of large gene lists using DAVID bioinformatics resources. Nat. Protoc 4, 44-57 (2009).

17. ENCODE Project Consortium. An integrated encyclopedia of DNA elements in the human genome. Nature 489, 57-74 (2012).

18. Pardiñas, A. F. et al. Common schizophrenia alleles are enriched in mutation-intolerant genes and in regions under strong background selection. Nat. Genet. 50, 381-389 (2018).

19. Wang, D. et al. Comprehensive functional genomic resource and integrative model for the human brain. Science, https://doi. org/10.1126/science.aat8464 (2018).

20. Fromer, M. et al. Gene expression elucidates functional impact of polygenic risk for schizophrenia. Nat. Neurosci. 19, 1442-1453 (2016).

21. Bai, Z. et al. Distinctive RNA expression profiles in blood associated with Alzheimer disease after accounting for white matter hyperintensities. Alzheimer Dis. Assoc. Disord. 28, 226-233 (2014).

22. Garcia-Esparcia, P., Hernández-Ortega, K., Ansoleaga, B., Carmona, M. \& Ferrer, I. Purine metabolism gene deregulation in Parkinson's disease. Neuropathol. Appl. Neurobiol. 41, 926-940 (2015).

23. Ma, Y. et al. Association of chromosome 5q21.3 polymorphisms with the exploratory eye movement dysfunction in schizophrenia. Sci. Rep. 5, 10299, https://doi.org/10.1038/srep10299 (2015).

24. Erickson-Ridout, K. K., Sun, D. \& Lazarus, P. Glucuronidation of the second-generation antipsychotic clozapine and its active metabolite N-desmethylclozapine. Potential importance of the UGT1A1 A(TA) ${ }_{7}$ TAA and UGT1A4 L48V polymorphisms. Pharmacogenet. Genomics 22, 561-576 (2012).

25. Corvin, A. \& Morris, D. W. Genome-wide association studies: findings at the major histocompatibility complex locus in psychosis. Biol. Psychiatry 75, 276-283 (2014).

26. Sanders, A. R. et al. Transcriptome study of differential expression in schizophrenia. Hum. Mol. Genet. 22, 5001-5014 (2013).

27. Sun, L. et al. Gene expression profiling of the xMHC region reveals 9 candidate genes in schizophrenia. J. Clin. Psychiatry 77, e597-599, https://doi.org/10.4088/JCP.15l10156 (2016).

28. Saradalekshmi, K. R. et al. DNA methyl transferase (DNMT) gene polymorphisms could be a primary event in epigenetic susceptibility to schizophrenia. PLoS One 9, e9818, https://doi.org/10.1371/journal.pone.0098182 (2014).

29. Chen, C. et al. Correlation between DNA methylation and gene expression in the brains of patients with bipolar disorder and schizophrenia. Bipolar Disord. 16, 790-799 (2014).

30. Håvik, B. et al. The complement control-related genes CSMD1 and CSMD2 associate to schizophrenia. Biol. Psychiatry 70, 35-42 (2011).

31. Sekar, A. et al. Schizophrenia risk from complex variation of complement component 4. Nature 530, 177-183 (2016).

32. Rubin, L. H. et al. Peripheral oxytocin and vasopressin are associated with clinical symptom severity and cognitive functioning in midlife women with chronic schizophrenia. Schizophr. Res. 195, 409-411 (2018).

33. Ansoleaga, B. et al. Decrease in olfactory and taste receptor expression in the dorsolateral prefrontal cortex in chronic schizophrenia. J. Psychiatr. Res. 60, 109-116 (2015).

34. Smeland, O. B. et al. Identification of genetic loci jointly influencing schizophrenia risk and the cognitive traits of verbal-numerical reasoning, reaction Time, and general cognitive function. JAMA Psychiatry 74, 1065-1075 (2017).

35. Dalman, C. et al. Signs of asphyxia at birth and risk of schizophrenia. Population-based case-control study. Br. J. Psychiatry 179, 403-408 (2001).

36. Altinoz, M. A. \& Ince, B. Hemoglobins emerging roles in mental disorders. Metabolical, genetical and immunological aspects. Int. J. Dev. Neurosci. 61, 73-85 (2017).

37. Richetto, J. et al. Genome-wide DNA methylation changes in a mouse model of infection-mediated neurodevelopmental disorders. Biol. Psychiatry 81, 265-276 (2017).

38. Tabarés-Seisdedos, R. et al. Evidence for association between structural variants in lissencephaly-related genes and executive deficits in schizophrenia or bipolar patients from a Spanish isolate population. Psychiatr. Genet. 18, 313-317 (2008).

39. Gao, J. et al. DNA methylation and gene expression of matrix metalloproteinase 9 gene in deficit and non-deficit schizophrenia. Front. Genet. 9, 646, https://doi.org/10.3389/fgene.2018.00646 (2018).

40. Mladinov, M. et al. Gene expression profiling of the dorsolateral and medial orbitofrontal cortex in schizophrenia. Transl. Neurosci. 7, 139-150 (2016).

41. Fazzari, P. et al. Cell autonomous regulation of hippocampal circuitry via Aph1b- $\gamma$-secretase/neuregulin 1 signalling. Elife 3 https:// doi.org/10.7554/eLife.02196 (2014).

42. Kim, S., Cho, H., Lee, D. \& Webster, M. J. Association between SNPs and gene expression in multiple regions of the human brain. Transl. Psychiatry 2, e113; 0.1038/tp.2012.42 (2012).

43. Passos Gregorio, S. et al. Analysis of coding-polymorphisms in NOTCH-related genes reveals NUMBL poly-glutamine repeat to be associated with schizophrenia in Brazilian and Danish subjects. Schizophr. Res. 88, 275-282 (2006).

44. Newman, M. E. J. \& Girvan, M. Finding and evaluating community structure in networks. Phys. Rev. E Stat. Nonlin. Soft Matter Phys. 69, $026113(2004)$

45. Su, G., Kuchinsky, A., Morris, J. H., States, D. J. \& Meng, F. GLay: community structure analysis of biological networks. Bioinformatics 26, 3135-3137 (2010).

46. Shannon, P. et al. Cytoscape: a software environment for integrated models of biomolecular interaction networks. Genome Res. 13 , 2498-2504 (2003).

\section{Acknowledgements}

The work of D.S.B. and D.J.B. were respectively supported by a Vice-Chancellor PhD bursary and Mathematics Summer Undergraduate Research Internship (MURS) at Nottingham Trent University (UK).

\section{Author contributions}

D.S.B., J.J.C. and N.C. conceived the research and wrote the manuscript. D.S.B., D.J.B. and N.C. performed data analysis. N.C. and J.J.C. directed the research. All authors reviewed manuscript.

\section{Competing interests}

The authors declare no competing interests. 


\section{Additional information}

Supplementary information is available for this paper at https://doi.org/10.1038/s41598-019-54514-2.

Correspondence and requests for materials should be addressed to N.C.

Reprints and permissions information is available at www.nature.com/reprints.

Publisher's note Springer Nature remains neutral with regard to jurisdictional claims in published maps and institutional affiliations.

(c) (i) Open Access This article is licensed under a Creative Commons Attribution 4.0 International

License, which permits use, sharing, adaptation, distribution and reproduction in any medium or format, as long as you give appropriate credit to the original author(s) and the source, provide a link to the Creative Commons license, and indicate if changes were made. The images or other third party material in this article are included in the article's Creative Commons license, unless indicated otherwise in a credit line to the material. If material is not included in the article's Creative Commons license and your intended use is not permitted by statutory regulation or exceeds the permitted use, you will need to obtain permission directly from the copyright holder. To view a copy of this license, visit http://creativecommons.org/licenses/by/4.0/.

(C) The Author(s) 2019 